\title{
Dosimetric comparison of two different applicators and rectal retraction methods used in inverse optimization-based intracavitary brachytherapy for cervical cancer
}

\author{
Fatih Biltekin, PhD, Melis Gultekin, MD, Melek Tugce Yilmaz, MD, Ferah Yildiz, MD \\ Department of Radiation Oncology, Faculty of Medicine, Hacettepe University, Ankara, Turkey
}

\begin{abstract}
Purpose: The purpose of this study was to evaluate the dosimetric differences between two different applicators and rectal-retraction methods used in image-guided brachytherapy (IGBT) for locally advanced cervical cancer (LACC).

Material and methods: Ten patients with LACC treated with definitive chemoradiotherapy and inverse optimization-based IGBT were included in this study. In each patient, at least one fraction of IGBT was performed using tandem-ovoids (TO) with vaginal gauze packing (VGP) or tandem-ring (TR) with rectal-retractor (RR). High-risk clinical target volume $\left(\mathrm{CTV}_{\mathrm{HR}}\right)$ and intermediate-risk CTV $\left(\mathrm{CTV}_{\mathrm{IR}}\right)$ were defined as CTVs, and bladder, rectum, sigmoid, small bowel, urethra, and vaginal mucosa were defined as organs at risk (OARs). All patients received 50.4 Gy external beam radiotherapy (EBRT) in 28 fractions. After EBRT, 28 Gy high-dose-rate (HDR) IGBT in 4 fractions was delivered to central disease. A plan comparison was performed using dose-volume histogram (DVH) and treatment planning parameters for CTVs and OARs.

Results: There were no significant differences in $\mathrm{D}_{90}$ values of $\mathrm{CTV}_{\mathrm{HR}}$. In terms of rectum dose, TR with RR was found to be significantly better than TO with VGP $\left(p<0.0001\right.$ for $\mathrm{D}_{2 \mathrm{~cm} 3}$ and $p<0.013$ for $\left.\mathrm{V}_{5 \mathrm{~Gy}}\right)$. Although, there were no statistically significant differences in $\mathrm{D}_{2 \mathrm{~cm} 3}$ value of bladder, sigmoid, small bowel, upper vaginal mucosa, and urethra, mean value of $\mathrm{D}_{2 \mathrm{~cm} 3}$ for all defined OARs were found lower in TR than in TO. Bladder $\mathrm{V}_{7 \mathrm{~Gy}}$, upper vaginal mucosa $\mathrm{V}_{7 \mathrm{~Gy}}$, middle and lower vaginal mucosa $\mathrm{D}_{2 \mathrm{~cm} 3}$ values were all found to be significantly lower for TR than for TO $(p<0.035)$. CTV $\mathrm{HR}_{\mathrm{HR}}$ and $\mathrm{CTV}_{\mathrm{IR}}$ volumes contoured in $\mathrm{TR}$ were approximately $11 \%$ and $9 \%$ smaller than $\mathrm{TO}$, respectively.

Conclusions: The results showed that there were no statistically differences in $D_{90}$ value of $C_{T V} V_{H R}$ and CTV $V_{I R}$. However, all DVH parameters for OARs in TR with RR were found to be better than in TO with VGP.
\end{abstract}

J Contemp Brachytherapy 2020; 12, 1: 35-43 DOI: https://doi.org/10.5114/jcb.2020.92699

Key words: cervix cancer, brachytherapy, applicator, rectal retraction methods.

\section{Purpose}

The standard of care for the treatment of locally advanced cervical cancer (LACC) is concurrent cisplatin-based chemoradiotherapy (CRT) and image-guided brachytherapy (IGBT) $[1,2,3,4]$. In radiation therapy (RT), local control and normal tissue complication probability is related to dose of radiation delivered to the target volume and organs at risk (OARs), respectively [5]. Dosimetric characteristics of IGBT makes it possible to deliver an ablative dose to the target volume while sparing surrounding OARs due to the steep dose gradient, which is not safely feasible with external beam radiotherapy (EBRT) techniques alone [6,7]. Therefore, IGBT has re- cently formed an integral part of the RT and it has been introduced to improve treatment outcomes in LACC $[8,9,10,11,12,13]$.

In the literature, Katz and Eifel [14], Perez et al. [15,16], and Viswanathan et al. [17] reported that local control and complication rates might be related with appropriate applicator selection and technical adequacy of the brachytherapy (BRT) implant. Therefore, selection of a suitable BRT applicator plays a crucial role to increase the treatment outcomes. In addition to applicator selection, treatment conditions like simulation or treatment protocol (rectum and bladder fullness), use of vaginal gauze packing (VGP), rectal spacer balloon (RSB), or rectal retractor (RR) significantly affect the quality of the treatment. 
According to the International Commission on Radiation Units and Measurements (ICRU) report 89 [18], there are various types of intracavitary applicators used in cervical cancer BRT, and most of these systems are composed of two main components including intrauterine tandem and vaginal applicator. The very first roots of intracavitary BRT applicators can be traced to radium based low-dose-rate (LDR) applications [19]. With the advance in technology, these applicators have evolved over many decades and they have been modified for ${ }^{137} \mathrm{Cs}$ and ${ }^{60} \mathrm{Co}$ artificial radioactive isotopes. Nowadays, ${ }^{192}$ Ir-based high-dose-rate (HDR) systems and BRT applicators compatible with these systems are widely used in clinics [18]. Although there are various type of intracavitary BRT applicators as defined in ICRU report 89 [18], tandem-ovoids (TO) and tandem-ring (TR) are the two most common used applicators in LACC $[20,21]$. Additionally, intracavitary BRT application requires an appropriate retraction method to place away rectum and bladder from radiation sources. In this way, complication probability for rectum and bladder can be reduced. Typically, VGP, RSB, and RR are the most common retraction methods used in IGBT for cervical cancer [22,23,24].

In the present study, volume optimization-based inverse planning techniques were used for both applicator geometries (TO and TR) and retraction methods (VGP and RR). Treatment planning parameters were then compared in terms of dose volume histogram (DVH) parameters, dose volume indices, clinical target volumes (CTVs), and calculated dwell time in $10 \mathrm{Ci}$ source activity. Although combined EBRT and BRT can result in significant urinary toxicity, urethra is not usually contoured as OAR, and there is a little existing data about urethral dose in the treatment of LACC. Therefore, in the present study, urethra was also contoured as an OAR and urethral dose was evaluated retrospectively. Additionally, upper, middle, and lower parts of the vaginal mucosa were contoured separately and doses were analyzed for each technique.

Table 1. FIGO stage distribution of the patients and included fractions of the treatment

\begin{tabular}{|c|c|c|}
\hline Patients & FIGO stage & $\begin{array}{c}\text { Fractions included } \\
\text { (TO-TR) }\end{array}$ \\
\hline 1 & IB2 & $1^{\text {st }}-2^{\text {nd }}$ \\
\hline 2 & IIB & $1^{\text {st }}-2^{\text {nd }}$ \\
\hline 3 & IIB & $1^{\text {st }}-2^{\text {nd }}$ \\
\hline 4 & IIB & $1^{\text {st }}-4^{\text {th }}$ \\
\hline 5 & IIB & $1^{\text {st }}-3^{\text {rd }}$ \\
\hline 6 & IB2 & $1^{\text {st }}-2^{\text {nd }}$ \\
\hline 7 & IIB & $1^{\text {st }}-2^{\text {nd }}$ \\
\hline 8 & IIB & $1^{\text {st }}-3^{\text {rd }}$ \\
\hline 9 & IB2 & $1^{\text {st }}-3^{\text {rd }}$ \\
\hline 10 & $\| I B$ & $1^{\text {st }}-3^{\text {rd }}$ \\
\hline
\end{tabular}

TO - tandem-ovoids, TR - tandem-ring

\section{Material and methods}

\section{Patients}

Ten patients with FIGO stage IB2 to IIIB cervical cancer, treated with concurrent CRT at our institute between July and November 2018 were included in this study. All patients' FIGO stage distribution is presented in Table 1. All patients received 50.4 Gy whole pelvic EBRT in 28 fractions, using volumetric modulated arc therapy (VMAT) with Elekta Versa HD linear accelerator (Elekta $\mathrm{AB}$, Stockholm, Sweden). Weekly cisplatin $40 \mathrm{mg} / \mathrm{m}^{2}$ concurrent with EBRT was administered to all patients. After EBRT treatment, 28 Gy HDR IGBT in 4 fractions were delivered to central disease with ${ }^{192}$ Ir sources using GammaMed Plus iX BRT unit (Varian Medical Systems, Palo Alto, CA). The study population was selected retrospectively among the patients who were topographically compatible with intracavitary BRT and treated with TO for one insertion and TR for another insertion. In this way, it was aimed to avoid patient-related differences like anatomical variations between two different patients.

\section{Intracavitary BRT application}

The intracavitary application was performed under conscious sedation in operating room conditions. Foley's catheter was inserted to obtain reproducible bladder fillings at the time of computed tomography (CT) simulation and during BRT treatment. For both TO and TR applications, the same tandem geometry, $6 \mathrm{~cm}$ tandem length and 45 degree tandem angle, were used to minimize uncertainties due to differences in tandem angle and length. The most commonly used sizes of the ovoid for TO applications were $2.0-2.5 \mathrm{~cm}$, and $5 \mathrm{~mm}$ build up cap was used in TR applicator. Anterior VGP was used to push the bladder and to stabilize applicator in both techniques. In TR applicator, RR was used as a retraction method and extra posterior VGP was not performed. In TO applicator, posterior VGP was carefully completed to displace the rectum away from the radioactive source, and at least 2-2.5 $\mathrm{m}$ packing gauze length was used for optimal packing.

\section{Simulation and treatment planning}

All patients underwent a CT scan using Toshiba Aquilion LB CT Simulator (Toshiba Medical Systems, Otowara, Japan) after every insertion with applicators in place. As a CT simulation protocol $100-120 \mathrm{kVp}$ tube voltage, 300-350 mAs current value and $2 \mathrm{~mm}$ slice thickness were used. After simulation processes, CT images were transferred to BrachyVision treatment planning system version 8.9 (TPS) (Varian Medical Systems, Palo Alto, CA) via digital imaging and communication in medicine (DICOM) connection.

Target volume determination was performed according to the gynecologic examination, and magnetic resonance imaging (MRI) findings at diagnosis and just before BRT treatment were completed following the recommendations of the Group Européen de Curiethérapie- 
European Society for Therapeutic Radiology and Oncology (GEC-ESTRO) $[25,26]$. According to the recommendations, high-risk (HR) and intermediate-risk (IR) CTVs were delineated. As for OARs, bladder, rectum, sigmoid, small bowel, and urethra were delineated [27], and vaginal mucosa including upper, middle and lower parts were contoured separately according to Westerveld et al.'s recommendations [28].

Treatment planning and dose calculation for the HDR BRT was performed using BrachyVision TPS. Volume optimization tool was used during the inverse optimization of the treatment plans, and similar optimization parameters were used to make it user-independent for both applicator geometries and retraction methods. Before starting the optimization, step size was set as $5 \mathrm{~mm}$ and the maximum active position range of the tandem for both TO and TR application was set as $7 \mathrm{~cm}$ from the tip of the tandem. For ovoids, the maximum active position range was set as $4 \mathrm{~cm}$ from the tip of the catheter and circular part was completely activated for ring applicator to mimic the standard loading pattern of manual optimization. Additionally, optimization parameters for inverse planning is presented in Table 2. As a clinical protocol, it was aimed to deliver at least 85 Gy (EBRT + BRT) equivalent dose in 2 Gy fractions $\left(\mathrm{EQD}_{2}\right)$ to $90 \%$ of $\mathrm{CTV}_{\mathrm{HR}}$ volume $\left(\mathrm{D}_{90}\right)$ and minimum $\mathrm{EQD}_{2}$ dose of $65 \mathrm{~Gy}$ to $90 \%$ of $\mathrm{CTV}_{\mathrm{IR}}$ volume $\left(\mathrm{D}_{90}\right)$. For OARs, the total dose in $\mathrm{D}_{2 \mathrm{~cm} 3}$ were limited to $90 \mathrm{~Gy}$ for bladder and $75 \mathrm{~Gy}$ for rectum, sigmoid, and small bowel. To calculate the total dose of EBRT plus BRT plans, linear quadratic model was applied with a reference dose rate of $0.5 \mathrm{~Gy} / \mathrm{h}$, a half-time repair of $1.5 \mathrm{~h}$, an $\alpha / \beta$ ratio of 10 Gy for CTVs, and 3 Gy for OARs.

A total of 20 fractions corresponding to one fraction TO and one fraction TR-based treatment planning for each patient were analyzed to evaluate the dosimetric differences between two different applicator geometries and rectal retraction methods. Fractions included in the BRT treatment are presented in Table 1 . In the analysis, DVH parameters of the $\mathrm{CTV}_{\mathrm{HR}}\left(\mathrm{D}_{90}\right.$ and $\left.\mathrm{D}_{98}\right), \mathrm{CTV}_{\mathrm{IR}}\left(\mathrm{D}_{90}\right.$ and $\left.\mathrm{D}_{98}\right)$, bladder $\left(\mathrm{D}_{2 \mathrm{~cm} 3}, \mathrm{D}_{0.1 \mathrm{~cm} 3}\right.$, and $\left.\mathrm{V}_{7 \mathrm{~Gy}}\right)$, rectum $\left(\mathrm{D}_{2 \mathrm{~cm} 3}\right.$, $\mathrm{D}_{0.1 \mathrm{~cm} 3}$, and $\left.\mathrm{V}_{5 \mathrm{~Gy}}\right)$, sigmoid $\left(\mathrm{D}_{2 \mathrm{~cm} 3}, \mathrm{D}_{0.1 \mathrm{~cm} 3}\right.$, and $\left.\mathrm{V}_{5 \mathrm{~Gy}}\right)$, small bowel $\left(D_{2 \mathrm{~cm} 3}, D_{0.1 \mathrm{~cm} 3}\right.$, and $\left.V_{5 G y}\right)$, urethra $\left(D_{2 \mathrm{~cm} 3}\right.$, $\mathrm{D}_{1 \mathrm{~cm} 3}$, and $\left.\mathrm{D}_{0.1 \mathrm{~cm} 3}\right)$, and vaginal mucosa $\left(\mathrm{D}_{2 \mathrm{~cm} 3}, \mathrm{D}_{0.1 \mathrm{~cm} 3}\right.$, $\mathrm{V}_{7 \mathrm{~Gy}}$, and $\mathrm{V}_{10 \mathrm{~Gy}}$ ) were noted for the comparison between TO with VGP and TR with RR applicators. Additionally, ICRU recto-vaginal point dose, vaginal reference point dose, differences in the target volumes, calculated total dwell times, weighting of the vaginal sources, and intrauterine sources in $10 \mathrm{Ci}$ source activity per fraction were analyzed for both applicator geometries and rectal retraction methods. Moreover, reference isodose volumes including TVDref, TV1.5Dref, TV2.0Dref, VDref, V1.5Dref, V2.0Dref, $\mathrm{V}_{60 \mathrm{~Gy}} \mathrm{EQD}_{2}$, and $\mathrm{V}_{85 \mathrm{~Gy}} \mathrm{EQD}_{2}$ were reported to evaluate the conformity of the treatment planning. TVDref, TV1.5Dref, and TV2.0Dref were the volumes of $\mathrm{CTV}_{\mathrm{HR}}$ receiving dose greater than or equal to $100 \%$, $150 \%$, and $200 \%$ of the prescription dose, respectively. Similarly, VDref, V1.5Dref, and V2.0Dref were the total volume receiving dose greater than or equal to $100 \%$, $150 \%$, and $200 \%$ of the prescription dose in external body, respectively.
Table 2. Optimization parameters for inverse planning

\begin{tabular}{lcc} 
Parameters & Optimization goals & Weighting \\
\hline CTV $_{\mathrm{HR}}$ & $\mathrm{D}_{95} \geq 700 \mathrm{cGy} / \mathrm{fr}$ & 100 \\
\cline { 2 - 3 } & $\mathrm{D}_{5} \leq 1,000 \mathrm{cGy} / \mathrm{fr}$ & 50 \\
\hline CTV $_{\mathrm{IR}}$ & $\mathrm{D}_{95} \geq 400 \mathrm{cGy} / \mathrm{fr}$ & 100 \\
\hline Bladder & $\mathrm{D}_{1 \mathrm{~cm} 3} \leq 500 \mathrm{cGy} / \mathrm{fr}$ & 75 \\
\hline Rectum & $\mathrm{D}_{1 \mathrm{~cm} 3} \leq 300 \mathrm{cGy} / \mathrm{fr}$ & 75 \\
\hline Sigmoid & $\mathrm{D}_{1 \mathrm{~cm} 3} \leq 300 \mathrm{cGy} / \mathrm{fr}$ & 75 \\
\hline Bowels & $\mathrm{D}_{1 \mathrm{~cm} 3} \leq 300 \mathrm{cGy} / \mathrm{fr}$ & 75 \\
\hline Upper vaginal mucosa & $\mathrm{D}_{1 \mathrm{~cm} 3} \leq 900 \mathrm{cGy} / \mathrm{fr}$ & 50 \\
\hline Normal tissue & $\mathrm{D}_{1 \mathrm{~cm} 3} \leq 1,000 \mathrm{cGy} / \mathrm{fr}$ & 50 \\
\hline Dwell time objective & Max $\leq 100 \mathrm{~s} / \mathrm{fr}$ & 75 \\
\cline { 2 - 3 } & $\mathrm{Min} \geq 1 \mathrm{~s} / \mathrm{fr}$ & 75
\end{tabular}

TO - tandem-ovoids, TR - tandem-ring, CTVHR - high-risk clinical target vol ume, CTVIR - intermediate-risk clinical target volume

\section{Statistical analysis}

All data were recorded and analyzed on Statistical Package for Social Sciences (SPSS) software (version 20, IBM). Descriptive statistics (mean and standard deviation) were calculated and unpaired Student's $t$-test was used to assess the relationship between the dosimetric values of two different applicators and retraction methods. $P<0.05$ was considered to be statistical significant.

\section{Results}

Dose-volume histogram parameters for CTVs and OARs are presented in Table $3 . \mathrm{D}_{90}$ and $\mathrm{D}_{98}$ value for $\mathrm{CTV}_{\mathrm{HR}}$ and $\mathrm{CTV}_{\mathrm{IR}}$ were found to be statistically similar for both applicators. Although, there were no statistical differences in bladder $\left(D_{2 \mathrm{~cm} 3}\right.$ and $\left.D_{0.1 \mathrm{~cm} 3}\right)$, sigmoid $\left(D_{2 \mathrm{~cm} 3}\right.$, $\mathrm{D}_{0.1 \mathrm{~cm} 3}$, and $\left.\mathrm{V}_{5 \mathrm{~Gy}}\right)$, small bowel $\left(\mathrm{D}_{2 \mathrm{~cm} 3}, \mathrm{D}_{0.1 \mathrm{~cm} 3}\right.$, and $\left.\mathrm{V}_{5 \mathrm{~Gy}}\right)$, upper vaginal mucosa $\left(\mathrm{D}_{2 \mathrm{~cm} 3}, \mathrm{D}_{0.1 \mathrm{~cm} 3}\right.$, and $\left.\mathrm{V}_{10 \mathrm{~Gy}}\right)$, and urethra $\left(D_{2 \mathrm{~cm} 3}, D_{1 \mathrm{~cm} 3}\right.$, and $\left.D_{0.1 \mathrm{~cm} 3}\right)$, mean value of these parameters for all defined OARs were found lower in TR with RR than in TO with VGP (Table 3). Moreover, bladder $\left(\mathrm{V}_{7 \mathrm{~Gy}}\right)$, upper vaginal mucosa $\left(\mathrm{V}_{7 \mathrm{~Gy}}\right)$, middle and lower vaginal mucosa $\left(\mathrm{D}_{2 \mathrm{~cm} 3}\right.$ and $\left.\mathrm{D}_{0.1 \mathrm{~cm} 3}\right)$ values were all found to be significantly lower for TR with RR than for TO with VGP $(p<0.045)$. In terms of rectal sparing, TR with RR was found to be significantly better than TO with VGP $\left(p<0.0001\right.$ for $\mathrm{D}_{2 \mathrm{~cm} 3}, p<0.000$ for $\mathrm{D}_{0.1 \mathrm{~cm} 3}$, and $p<0.013$ for $\left.\mathrm{V}_{5 \mathrm{~Gy}}\right)$. Similarly, as presented in Table 4, ICRU recto-vaginal reference point dose were found to be statistically lower for TR with RR than for TO with VGP. However, there were no statistically significant differences in ICRU vaginal reference point doses for both applications (Table 4). In addition to DVH parameters, volumes of CTVs and total dwell times in $10 \mathrm{Ci}$ source activities are shown in Table 5. CTV $\mathrm{HR}_{\mathrm{H}}$ and $\mathrm{CTV}_{\mathrm{IR}}$ volumes contoured in TR-based applications were approximately $11 \%$ and $9 \%$ smaller than TO-based applications, respectively. The 
Table 3. Comparison of DVH parameters for TO with VGP and TR with RR

\begin{tabular}{|c|c|c|c|c|}
\hline DVH parameters & $\begin{array}{c}\text { TO } \\
(\text { Mean } \pm \text { SD })\end{array}$ & $\begin{array}{c}\text { TR } \\
(\text { Mean } \pm S D)\end{array}$ & $\begin{array}{c}\Delta \text { Mean } \pm \text { SD } \\
(\text { TO-TR })^{*}\end{array}$ & $p$ value \\
\hline $\mathrm{CTV}_{\mathrm{HR}} \mathrm{D}_{90}$ (cGy) & $706.2 \pm 4.5$ & $710.6 \pm 5.5$ & $-4.4 \pm 6.4$ & 0.0647 \\
\hline $\mathrm{CTV}_{\mathrm{HR}} \mathrm{D}_{98}$ (cGy) & $574.5 \pm 27.8$ & $595.3 \pm 16.6$ & $-20.8 \pm 34.8$ & 0.058 \\
\hline $\mathrm{CTV}_{\mathbb{I R}} \mathrm{D}_{90}$ (cGy) & $510.8 \pm 32.2$ & $497.3 \pm 37.9$ & $13.5 \pm 38.8$ & 0.402 \\
\hline $\mathrm{CTV}_{\mathbb{I R}} \mathrm{D}_{98}$ (cGy) & $398.4 \pm 28.4$ & $384.6 \pm 44.8$ & $13.8 \pm 53.5$ & 0.422 \\
\hline Rectum $\mathrm{D}_{2 \mathrm{~cm}^{3}}(\mathrm{cGy})$ & $493.7 \pm 60.0$ & $357.4 \pm 62.9$ & $136.3 \pm 40.2$ & 0.0001 \\
\hline Rectum $\mathrm{D}_{0.1 \mathrm{~cm}^{3}}$ (cGy) & $658.2 \pm 67.6$ & $493.7 \pm 87.9$ & $164.5 \pm 79.7$ & 0.0002 \\
\hline Rectum $V_{5 G y}\left(\mathrm{~cm}^{3}\right)$ & $2.2 \pm 1.6$ & $0.2 \pm 0.4$ & $2.0 \pm 1.3$ & 0.0013 \\
\hline Bladder $\mathrm{D}_{2 \mathrm{~cm} 3^{3}}$ & $585.3 \pm 134.1$ & $490.8 \pm 97.2$ & $94.5 \pm 93.6$ & 0.088 \\
\hline Bladder $\mathrm{D}_{0.1 \mathrm{~cm}^{3}}(\mathrm{cGy})$ & $726.1 \pm 67.6$ & $670.2 \pm 87.9$ & $55.9 \pm 272.2$ & 0.582 \\
\hline Bladder $V_{7 G y}\left(\mathrm{~cm}^{3}\right)$ & $1.0 \pm 0.9$ & $0.3 \pm 0.5$ & $0.7 \pm 1.0$ & 0.035 \\
\hline Small bowels $D_{2 \mathrm{~cm}^{3}}(\mathrm{cGy})$ & $356.4 \pm 172.8$ & $322.7 \pm 146.9$ & $33.7 \pm 132.8$ & 0.644 \\
\hline Small bowels $\mathrm{D}_{0.1 \mathrm{~cm}^{3}}$ (cGy) & $498.2 \pm 217.9$ & $443.5 \pm 215.9$ & $54.7 \pm 170.0$ & 0.580 \\
\hline Small bowels $V_{5 G y}\left(\mathrm{~cm}^{3}\right)$ & $1.6 \pm 2.5$ & $0.6 \pm 0.9$ & $1.0 \pm 2.0$ & 0.206 \\
\hline Sigmoid $\mathrm{D}_{2 \mathrm{~cm}^{3}}(\mathrm{cGy})$ & $323.9 \pm 193.9$ & $265.1 \pm 134.3$ & $58.8 \pm 169.5$ & 0.441 \\
\hline Sigmoid $\mathrm{D}_{0.1 \mathrm{~cm}^{3}}(\mathrm{cGy})$ & $420.3 \pm 251.5$ & $316.6 \pm 179.7$ & $103.7 \pm 276.0$ & 0.303 \\
\hline Sigmoid $\mathrm{V}_{5 \mathrm{~Gy}}\left(\mathrm{~cm}^{3}\right)$ & $1.3 \pm 2.1$ & $0.3 \pm 0.7$ & $1.0 \pm 1.7$ & 0.159 \\
\hline Upper vaginal mucosa $\mathrm{D}_{2 \mathrm{~cm}^{3}}$ (cGy) & $798.7 \pm 158.5$ & $656.5 \pm 208.6$ & $142.2 \pm 173.7$ & 0.103 \\
\hline Upper vaginal mucosa $\mathrm{D}_{0.1 \mathrm{~cm}^{3}}$ (cGy) & $1217.0 \pm 425.1$ & $1179.0 \pm 456.2$ & $38.0 \pm 108.1$ & 0.85 \\
\hline Upper vaginal mucosa $V_{7 G y}\left(\mathrm{~cm}^{3}\right)$ & $2.9 \pm 2.2$ & $1.2 \pm 0.9$ & $1.7 \pm 2.1$ & 0.0318 \\
\hline Upper vaginal mucosa $\mathrm{V}_{10 \mathrm{~Gy}}\left(\mathrm{~cm}^{3}\right)$ & $0.6 \pm 0.8$ & $0.4 \pm 0.4$ & $0.2 \pm 0.9$ & 0.409 \\
\hline Middle vaginal mucosa $\mathrm{D}_{2 \mathrm{~cm}^{3}}$ (cGy) & $380.9 \pm 141.8$ & $233.4 \pm 102.5$ & $147.5 \pm 76.9$ & 0.0158 \\
\hline Middle vaginal mucosa $\mathrm{D}_{0.1 \mathrm{~cm}^{3}}$ (cGy) & $617.9 \pm 296.8$ & $343.8 \pm 149.9$ & $274.1 \pm 185.3$ & 0.018 \\
\hline Lower vaginal mucosa $\mathrm{D}_{2 \mathrm{~cm}^{3}}$ (cGy) & $147.7 \pm 59.5$ & $97.8 \pm 30.3$ & $49.9 \pm 43.1$ & 0.0153 \\
\hline Lower vaginal mucosa $\mathrm{D}_{0.1 \mathrm{~cm}^{3}}$ (cGy) & $196.0 \pm 104.6$ & $119.6 \pm 40.2$ & $76.4 \pm 78.0$ & 0.045 \\
\hline Urethra $D_{2 \mathrm{~cm}^{3}}(\mathrm{cGy})$ & $50.8 \pm 50.0$ & $31.1 \pm 34.8$ & $19.7 \pm 27.2$ & 0.32 \\
\hline Urethra $D_{1 \mathrm{~cm}^{3}}(\mathrm{cGy})$ & $144.3 \pm 53.2$ & $95.2 \pm 68.8$ & $49.1 \pm 53.8$ & 0.0911 \\
\hline Urethra $\mathrm{D}_{0.1 \mathrm{~cm}^{3}}$ (cGy) & $296.7 \pm 90.0$ & $213.0 \pm 88.8$ & $83.7 \pm 53.0$ & 0.051 \\
\hline
\end{tabular}

${ }^{*} \Delta$ Mean $(T O-T R)^{*}$ - mean value of the difference, TO - tandem-ovoids, TR - tandem-ring, CTVHR-high-risk clinical target volume, CTVIR - intermediate-risk clinical target volume

Table 4. ICRU recto-vaginal and vaginal reference point dose for both TO with VGP and TR with RR

\begin{tabular}{lcccc} 
Planning parameters & TO $($ Mean \pm SD) & TR $($ Mean \pm SD) & $\begin{array}{c}\Delta \text { Mean } \pm \text { SD } \\
(\text { TO-TR)* }\end{array}$ & $p$ value \\
\hline Recto-vaginal point dose (cGy) & $465.7 \pm 47.3$ & $360.1 \pm 49.5$ & $105.6 \pm 57.0$ & 0.0001 \\
\hline Vaginal point dose left (cGy) & $777.5 \pm 209.2$ & $705.2 \pm 145.6$ & $72.3 \pm 113.3$ & 0.382 \\
\hline Vaginal point dose right (cGy) & $745.4 \pm 131.1$ & $725.5 \pm 142.9$ & $-19.9 \pm 233.4$ & 0.749
\end{tabular}

${ }^{*} \Delta$ Mean $(T O-T R)^{*}-$ mean value of the difference, $T O$ - tandem-ovoids, $T R$ - tandem-ring 
Table 5. Target volumes and total dwell times in $10 \mathrm{Ci}$ source activity for TO and TR applicators

\begin{tabular}{lcccc} 
DVH parameters & $\begin{array}{c}\text { TO } \\
(\text { Mean } \pm \text { SD })\end{array}$ & $\begin{array}{c}\text { TR } \\
(\text { Mean } \pm \text { SD })\end{array}$ & $\begin{array}{c}\Delta \text { Mean } \pm \text { SD } \\
(\text { TO-TR })^{*}\end{array}$ & $p$ value \\
\hline CTV $_{\text {HR }}$ volume $\left(\mathrm{cm}^{3}\right)$ & $32.8 \pm 10.3$ & $29.4 \pm 8.0$ & $3.4 \pm 9.0$ & 0.424 \\
\hline CTV $_{\mathbb{I R}}$ volume $\left(\mathrm{cm}^{3}\right)$ & $77.4 \pm 30.1$ & $70.8 \pm 30.5$ & $6.6 \pm 13.9$ & 0.633 \\
\hline Total dwell time $(\mathrm{s})$ & $386.0 \pm 80.6$ & $302.4 \pm 67.7$ & $83.6 \pm 65.9$ & 0.0218
\end{tabular}

${ }^{*} \Delta$ Mean $(T O-T R)^{*}$ - mean value of the difference, TO - tandem-ovoids, TR - tandem-ring, CTV ${ }_{H R}$ - high-risk clinical target volume, $C T V_{I R}-$ intermediate-risk clinical target volume

typical view of delineated CTVs and dose distributions of both applications are illustrated in Figures 1 and 2, respectively. When the total irradiation time per fraction was calculated, mean dwell time in $10 \mathrm{Ci}$ source activity for TO and TR applicators were found as $386 \mathrm{~s}$ and $302.4 \mathrm{~s}$, respectively $(p<0.0218)$; weighting of the vaginal and intrauterine sources for each patient are presented in Table 6. Additionally, TR with RR was found to be statistically superior than TO with VGP in terms of VDref, $\mathrm{V}_{60 G y} \mathrm{EQD}_{2}$ and $\mathrm{V}_{85 \mathrm{~Gy}} \mathrm{EQD}_{2}$ as presented in Table 7 .

\section{Discussion}

The definitive treatment of LACC includes both pelvic EBRT with concomitant platinum-based chemotherapy and BRT boost to the central disease. TO and TR are the two most common intracavitary BRT applicators used in the treatment of LACC $[1,2,3,4,18,20,21]$. TO applicator is the variation of traditional Manchester, Fletcher, Henschke systems, and TR applicator is derived from the Stockholm system [18]. TR applicators have recently come into widespread use in IGBT of cervical cancer due to the easiness of application compared to TO applicators. Additionally, it has a predictable and fixed geometry $[20,21,29]$. All these physical properties of TR applicator make it more advantageous in clinical use. Although TR applicator can be used in all patient groups requiring intracavitary BRT, patients with non-bulky disease, superficial or obliterated vaginal fornix, or narrow vaginal cavity are the ideal patients' group in cervical cancer $[20,21,29]$. In addition to applicator geometries, a retraction method play an important role to place away rectum from radiation source and to increase the conformity of the BRT treatment plan [22].

In the literature, the dosimetric comparison of different applicator geometries (TO and TR) and rectal retraction methods (RR, VGP, and tandem Foley balloon) have been studied. Ma et al. [20], Rangarajan [29], and Erickson et al. [30] reported higher rectal dose in TO compared with TR applicator. In the study performed by Rangarajan [29], it was pointed out that in addition to posterior VGP, RR was used in all TR applications and lower rectum dose in TR applications was attributed to the use of RR. Additionally, Kong et al. [22] compared three rectal retraction methods including RR blade, VGP, and a tandem Foley balloon. They discovered that RR was more advantageous in terms of rectal dose compared to other defined methods. In the present study, TR applicator with RR was also found to be statistically superior to TO applicator with posterior VGP in terms of rectal sparing.

In terms of bladder doses, Ma et al. [20] and Rangarajan [29] reported that there were no significant differences between TO and TR for $\mathrm{D}_{2 \mathrm{~cm} 3}$ value. Similarly, statistically significant difference was not found for the same DVH parameter in the present study. However, there was a slight trend towards increased $\mathrm{D}_{2 \mathrm{~cm} 3}$ value, with TO and $V_{7 G y}$ for bladder founded to be significantly higher in TO compared with TR-based treatment plans.

In addition to rectum and bladder dose, in the present study, upper, middle, lower vaginal mucosa, and urethral dose were evaluated. Although vaginal mucosa and urethra have been considered as radio-resistant organs, recent studies have reported a higher incidence of late vaginal toxicity (e.g. vaginal shortening and dyspareunia)
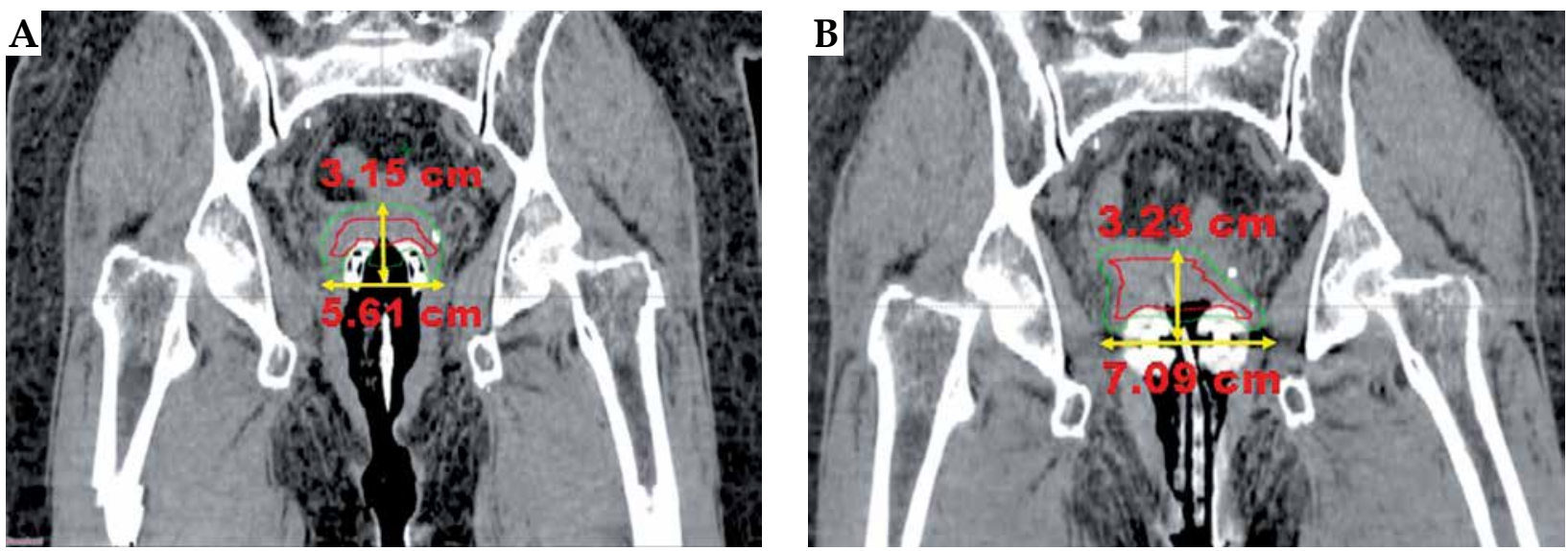

Fig. 1. A typical view of delineated CTVHR (red) and CTVIR (green) volume in coronal image section for A) TR and B) TO applicators on a same patient 

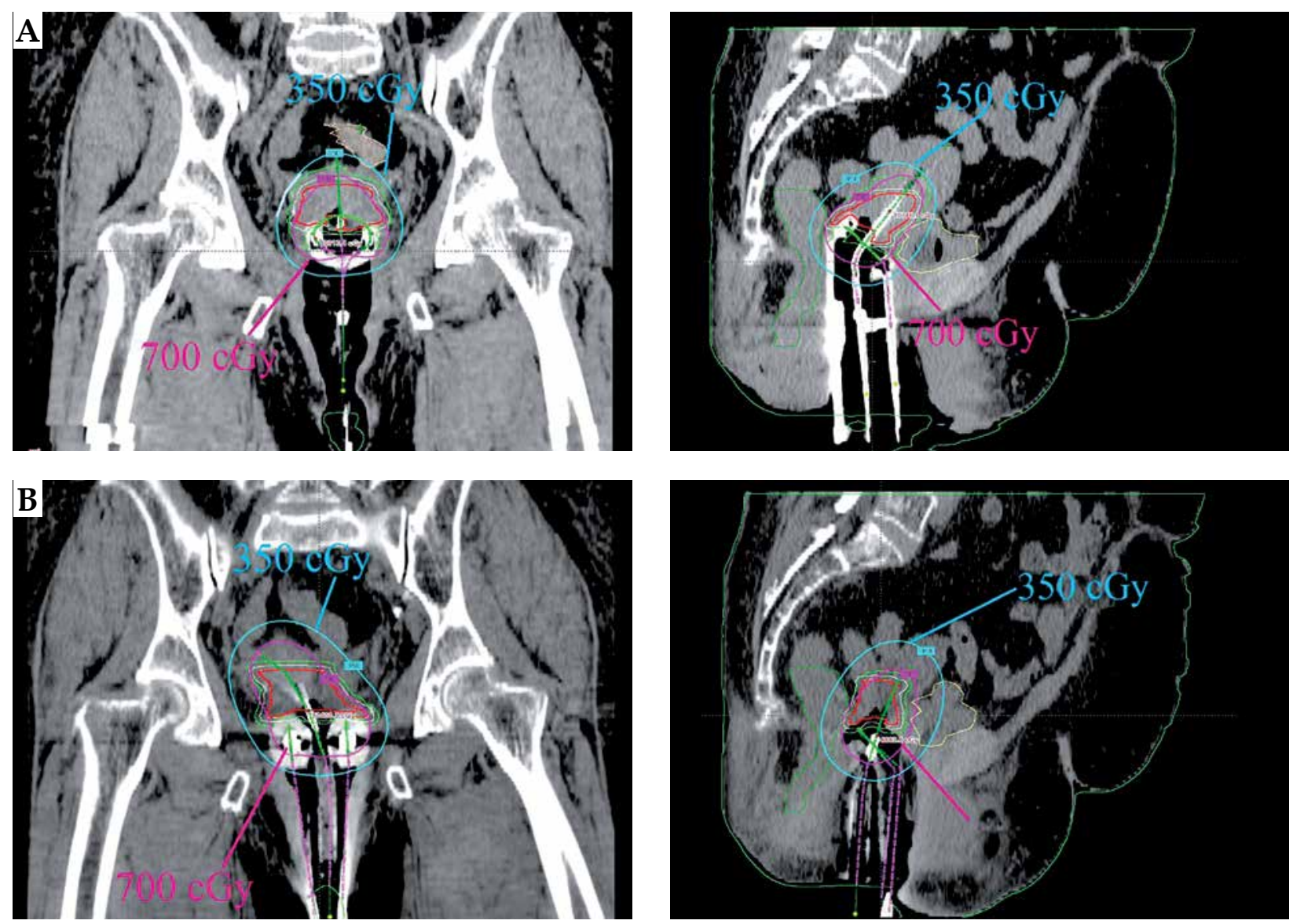

Fig. 2. Dose distributions in coronal and sagittal image sections using A) TR and B) TO applicators on the same patient

and severe urethral toxicity (e.g. urethral necrosis) in the treatment of gynecological patients with combined treatment of EBRT and BRT [31,32]. However, so far, there is a little existing data about dose tolerance limits for vaginal mucosa and urethral dose in the treatment of LACC. To the best of our knowledge, this is the first comprehen-

Table 6. Weighting of the vaginal and intrauterine sources in inverse optimization-based planning

\begin{tabular}{lcccc} 
& & TO $(\mathrm{s})$ & & TR (s) \\
\cline { 2 - 5 } Patient & $\mathrm{T}$ & $\mathrm{O}$ & $\mathrm{T}$ & 193 \\
\hline 1 & 281.2 & 124.6 & 124.3 & 217.4 \\
\hline 2 & 275.7 & 117.8 & 104.6 & 158.3 \\
\hline 3 & 193 & 136 & 172.6 & 123.6 \\
\hline 5 & 169.7 & 147.8 & 100.5 & 222.1 \\
\hline 6 & 156.2 & 133.2 & 110.5 & 223.9 \\
\hline 7 & 143.5 & 358.6 & 49.6 & 242.6 \\
\hline 9 & 280.3 & 164.3 & 210.3 & 200.3 \\
\hline 10 & 199.5 & 253.6 & 97.4 & 102.2 \\
\hline Mean (s) & 200.2 & 258 & 117 & 187.5 \\
\hline Weighting of the source & 147.1 & 120 & 114.9 & $62.0 \%$
\end{tabular}

TO - tandem-ovoids, TR-tandem-ring 
Table 7. Reference dose volumes in CTV $\mathrm{HR}_{\mathrm{R}}$ and external body for both TO with VGP and TR with RR

\begin{tabular}{|c|c|c|c|c|}
\hline DVH parameters & TO $($ Mean \pm SD) & TR (Mean \pm SD) & $\begin{array}{c}\Delta \text { Mean } \pm \text { SD } \\
\text { (TO-TR) }^{*}\end{array}$ & $p$ value \\
\hline VDref $\left(\mathrm{cm}^{3}\right)$ & $84.7 \pm 26.3$ & $61.4 \pm 20.2$ & $23.3 \pm 22.4$ & 0.039 \\
\hline TVDref $\left(\mathrm{cm}^{3}\right)$ & $29.6 \pm 9.3$ & $26.7 \pm 7.1$ & $2.9 \pm 8.1$ & 0.442 \\
\hline V1.5Dref $f^{\star \star}\left(\mathrm{cm}^{3}\right)$ & $44.2 \pm 14.2$ & $32.9 \pm 11$ & $11.3 \pm 13.5$ & 0.063 \\
\hline TV1.5Dref $\left(\mathrm{cm}^{3}\right)$ & $19.0 \pm 7.1$ & $16.5 \pm 5.1$ & $2.5 \pm 6.7$ & 0.376 \\
\hline V2.0Dref** $\left(\mathrm{cm}^{3}\right)$ & $26.3 \pm 9.2$ & $19.8 \pm 6.9$ & $6.6 \pm 9.1$ & 0.087 \\
\hline TV2.0Dref $\left(\mathrm{cm}^{3}\right)$ & $12.1 \pm 5.0$ & $9.9 \pm 3.9$ & $2.2 \pm 4.9$ & 0.294 \\
\hline$V_{60 G y} E Q D_{2}\left(\mathrm{~cm}^{3}\right)$ & $380.8 \pm 114.8$ & $267.6 \pm 89.8$ & $113.2 \pm 88.9$ & 0.024 \\
\hline$V_{85 G y} E Q D_{2}\left(\mathrm{~cm}^{3}\right)$ & $95 \pm 29.4$ & $68.6 \pm 22.6$ & $26.4 \pm 24.2$ & 0.037 \\
\hline
\end{tabular}

${ }^{*} \Delta$ Mean $(T O-T R)^{*}$ - mean value of the difference, ${ }^{* *}$ V1.5Dref and V2.0Dref are the volumes of external body receiving $150 \%$ and $200 \%$ of the prescription dose, respectively, TO -tandem-ovoids, TR - tandem-ring, VDref-volume receiving dose greater than or equal to the prescribed reference dose in external body, TVDrefvolume of HR-CTV receiving dose greater than or equal to the prescribed reference dose, V1.5Dref-volumes of external body receiving $150 \%$ of the prescription dose, TV1.5Dref - volumes of HR-CTV receiving 150\% of the prescription dose, V2.0Dref-volumes of external body receiving 200\% of the prescription dose, TV2.0Drefvolumes of HR-CTV receiving $200 \%$ of the prescription dose, $V_{60 G y} E Q D_{2}-$ isodose surface volume of 60 Gy equivalent dose in $2 G y$ per fraction, $V_{80 G y} E Q D_{2}-i s o d o s e$ surface volume of 85 Gy equivalent dose in 2 Gy per fraction

sive study to evaluate urethral dose and vaginal mucosa dose including upper, middle, and lower parts separately for both applicator geometries. Although, in the literature it was stated that vaginal mucosa dose might be higher in TR applicators compared with TO due to the smaller thickness of the build-up material [30,33,34], in the present study, vaginal mucosa dose was found lower in TR with RR than in TO with VGP. In fact, significant differences were demonstrated for upper vaginal mucosa $\left(\mathrm{V}_{7 \mathrm{~Gy}}\right)$, and middle and lower vaginal mucosa $\left(\mathrm{D}_{2 \mathrm{~cm} 3}\right.$ and $\left.\mathrm{D}_{0.1 \mathrm{~cm} 3}\right)$ values. This can be attributed to the fact that total volume receiving dose greater than or equal to the prescribed reference dose in external body was found to be higher in TO than in TR applicator.

Ma et al. [20] and Levin et al. [21] also reported that contoured target volumes were smaller in TR compared to TO applicators. Similar differences were observed in the present study, and the differences in delineated volumes can be attributed to the geometric design of TO applicator, allowing for wider transverse displacement of the $\mathrm{CTV}_{\mathrm{HR}}$ and $\mathrm{CTV}_{\mathrm{IR}}$ volumes at the level of the ovoids, when compared with ring applicator. Additionally, in the study performed by Ma et al. [20] and Levin et al. [21], the dose prescription was performed according to reference point A as defined in ICRU 38 [35]. However, in the present study, the reference dose was prescribed to $D_{90}$ value of $\mathrm{CTV}_{\mathrm{HR}}$ and $\mathrm{CTV}_{\mathrm{IR}}$, as recommended in GEC-ESTRO guideline [26]. Additionally, the comparison of different applicator geometries and retraction methods in the same patient anatomy can be shown as one of the major advantage of the present study to minimize the effects of anatomical differences between two patient groups.

In terms of irradiation time, similar to Ma et al. [20] and Levin et al. [21], total dwell times for TR applicator were found lower than that for TO applicator. This difference could be attributed to the geometry of intravaginal component of the BRT applicators. According to our analysis, in TO applicator weighting of the intrauterine source was found higher than intravaginal source during the irradiation of the anterior part of target volume. In contrast to TO applicator, the weighting of the intravaginal source was found higher than intrauterine source in TR applicator during the irradiation of related part of the target volume. This could be related to the fact that the upper part of intravaginal component in TR applicator was so much closer to the defined region than that of both intravaginal and intrauterine component in TO applicator. Due to the closer source position to the target volume, total dwell times were also found lesser in TR applicator than that in TO applicator. Another noteworthy point is that in the present study, volume-based optimization method has been validated for both applicator geometries (TO with VGP and TR with RR) in defined conditions. Similarly, in the literature, Jamema et al. [36] and Kannan et al. [37] reported that inverse planning could offer good sparing of critical structures without compromising the target coverage compared to standard techniques, and so it could improve the quality of treatment plans in intracavitary BRT. Nevertheless, as stated by Chajon et al. [38], straightforward use of inverse optimization might generated significant heterogeneity in dwell times, and loading pattern of the source in inverse optimization could be completely different from the standard loading pattern. Therefore, inverse optimization protocols should be validated before implementation in clinical practice and the loading pattern of the source should be verified after each optimization, since it requires extra caution to test the dosimetrical relevance of new optimization techniques in intracavitary BRT modalities. In the present study, loading pattern of the intrauterine and intravaginal sources for all treatment plans were also validated using manual planning technique, and it was found almost similar for both manual planning (54.5-45.5\% for TO and 40.1-59.9\% for TR) and inverse optimization-based planning (53.0$47.0 \%$ for TO and $38.0-62.0 \%$ for TR).

This study has also some limitations that have to be pointed out. The first limitation is that in TR applicator, $\mathrm{RR}$ was used as a retraction method and extra packing 
procedure was not performed. However, in TO applicator, VGP was performed due to the fact that there was no rectal retractor integrated into the existing system. Therefore, in terms of rectal sparing, two different applicator geometries with different retraction methods including TO with VGP and TR with RR were analyzed together. Another limitation is that this study focused only on treatment planning parameters of different applicator geometries and retraction methods. However, the effectiveness of the systems or applicator geometries was not evaluated in terms of short- and long-term clinical outcomes. Therefore, clinical studies need to be carried out to evaluate the correlations between dosimetric and clinical parameters for defined applicator geometries and retraction methods.

\section{Conclusions}

The results showed that there were no statistical differences in $\mathrm{D}_{90}$ value of $\mathrm{CTV}_{\mathrm{HR}}$ and $\mathrm{CTV}_{\mathrm{IR}}$. However, all DVH parameters for OARs in TR were found to be better than in TO applicators. In terms of rectum dose, TR with RR provides statistically better rectal sparing than TO with posterior VGP. Nevertheless, short- and longterm clinical impact of the dosimetric differences for two different applicator geometries and retraction methods needs to be evaluated further.

\section{Disclosure}

The authors report no conflict of interest.

\section{References}

1. Rose PG, Bundy BN, Watkins EB et al. Concurrent cisplatin-based radiotherapy and chemotherapy for locally advanced cervical cancer. N Engl J Med 1999; 340: 1144-1153.

2. Thomas GM. Improved treatment for cervical cancer - concurrent chemotherapy and radiotherapy. N Engl J Med 1999; 340: 1198-1200.

3. Stehman FB, Ali S, Keys HM et al. Radiation therapy with or without weekly cisplatin for bulky stage $1 \mathrm{~B}$ cervical carcinoma: follow-up of a Gynecologic Oncology Group trial. Am J Obstet Gynecol 2007; 197: 503.e1-6.

4. Tanderup K, Eifel PJ, Yashar CM et al. Curative radiation therapy for locally advanced cervical cancer: brachytherapy is NOT optional. Int J Radiat Oncol Biol Phys 2014; 88: 537-539.

5. Nesvacil N, Tanderup K, Lindegaard JC et al. Can reduction of uncertainties in cervix cancer brachytherapy potentially improve clinical outcome? Radiother Oncol 2016; 120: 390396.

6. Akiyama H, Pesznyák C, Béla D et al. Image guided highdose-rate brachytherapy versus volumetric modulated arc therapy for head and neck cancer: A comparative analysis of dosimetry for target volume and organs at risk. Radiat Oncol 2018; 52: 461-467.

7. Weiner AA, Schwarz JK. Intracavitary brachytherapy for gynecologic malignancies: applications and innovations. Mo Med 2015; 112: 366-372.

8. Cho O, Chun M. Management for locally advanced cervical cancer: new trends and controversial issues. Radiat Oncol J 2018; 36: 254-264.

9. Pötter R, Georg P, Dimopoulos JC et al. Clinical outcome of protocol based image (MRI) guided adaptive brachytherapy combined with 3D conformal radiotherapy with or without chemotherapy in patients with locally advanced cervical cancer. Radiother Oncol 2011; 100: 116-123.

10. Charra-Brunaud C, Harter V, Delannes M et al. Impact of 3D image-based PDR brachytherapy on outcome of patients treated for cervix carcinoma in France: results of the French STIC prospective study. Radiother Oncol 2012; 103: 305-313.

11. Ribeiro I, Janssen H, De Brabandere $M$ et al. Long term experience with 3D image guided brachytherapy and clinical outcome in cervical cancer patients. Radiother Oncol 2016; 120 : 447-454.

12. Sturdza A, Pötter R, Fokdal LU et al. Image guided brachytherapy in locally advanced cervical cancer: Improved pelvic control and survival in RetroEMBRACE, a multicenter cohort study. Radiother Oncol 2016; 120: 428-433.

13. Tanderup K, Fokdal LU, Sturdza A et al. Effect of tumor dose, volume and overall treatment time on local control after radiochemotherapy including MRI guided brachytherapy of locally advanced cervical cancer. Radiother Oncol 2016; 120 : 441-446.

14. Katz A, Eifel PJ. Quantification of intracavitary brachytherapy parameters and correlation with outcome in patients with carcinoma of the cervix. Int J Radiat Oncol Biol Phys 2000; 48: 1417-1425.

15. Perez CA, Breaux S, Madoc-Jones $\mathrm{H}$ et al. Radiation therapy alone in the treatment of carcinoma of uterine cervix. I. Analysis of tumor recurrence. Cancer 1983; 5: 1393-1402.

16. Perez CA, Breaux S, Bedwinek JM et al. Radiation therapy alone in the treatment of carcinoma of the uterine cervix. II. Analysis of complications. Cancer 1984; 54: 235-246.

17. Viswanathan AN, Moughan J, Small W et al. The quality of cervical cancer brachytherapy implantation and the impact on local recurrence and disease-free survival in radiation therapy oncology group prospective trials 0116 and 0128 . Int J Gynecol Cancer 2012; 22: 123-131.

18. Potter R, Kirisits C, Erickson C et al. Prescribing, recording, and reporting brachytherapy for cancer of the cervix. J ICRU 2013; 13: NP.

19. Mould RF. The historical roots of modern brachytherapy for cervical and endometrial cancer. In Radiation Oncology of Gynecological Cancers. Springer, Berlin, Heidelberg 1977; 1-9.

20. Ma JK, Mourad WF, Allbright R et al. Short-term clinical outcome and dosimetric comparison of tandem and ring versus tandem and ovoids intracavitary applicators. J Contemp Brachytherapy 2015; 7: 218-223.

21. Levin D, Menhel J, Rabin T et al. Dosimetric comparison of tandem and ovoids vs. tandem and ring for intracavitary gynecologic applications. Med Dosim 2008; 33: 315-320.

22. Kong I, Vorunganti S, Patel $\mathrm{M}$ et al. Prospective comparison of rectal dose reduction during intracavitary brachytherapy for cervical cancer using three rectal retraction techniques. Brachytherapy 2016; 15: 450-455.

23. Rai B, Patel FD, Chakraborty $S$ et al. Bladder-rectum spacer balloon in high-dose-rate brachytherapy in cervix carcinoma. Int J Radiat Oncol Biol Phys 2013; 85: e217-e222.

24. Rockey WM, Bhatia SK, Jacobson GM et al. The dosimetric impact of vaginal balloon-packing on intracavitary highdose-rate brachytherapy for gynecological cancer. J Contemp Brachytherapy 2013; 5: 17-22.

25. Haie-Meder C, Pötter R, Van Limbergen E et al. Recommendations from Gynaecological (GYN) GEC-ESTRO Working Group (I): concepts and terms in 3D image based 3D treatment planning in cervix cancer brachytherapy with emphasis on MRI assessment of GTV and CTV. Radiother Oncol 2005; 74: 235-245.

26. Pötter R, Haie-Meder C, Van Limbergen E et al. Recommendations from gynaecological (GYN) GEC ESTRO working 
group (II): concepts and terms in 3D image-based treatment planning in cervix cancer brachytherapy $3 \mathrm{D}$ dose volume parameters and aspects of 3D image-based anatomy, radiation physics, radiobiology. Radiother Oncol 2006; 78: 67-77.

27. Gay HA, Barthold HJ, O'Meara E et al. Pelvic normal tissue contouring guidelines for radiation therapy: a Radiation Therapy Oncology Group consensus panel atlas. Int J Radiat Oncol Biol Phys 2012; 83: e353-e362.

28. Westerveld H, Pötter R, Berger D et al. Vaginal dose point reporting in cervical cancer patients treated with combined 2D/3D external beam radiotherapy and 2D/3D brachytherapy. Radiother Oncol 2013; 107: 99-105.

29. Rangarajan R. Dosimetric evaluation of image based brachytherapy using tandem ovoid and tandem ring applicators. Rep Pract Oncol Radiother 2018; 23: 57-60.

30. Erickson B, Jones R, Rownd J et al. Is the tandem and ring applicator a suitable alternative to the high dose rate selectron tandem and ovoid applicator? J Brachytherapy Int 2000; 16: 131-144.

31. Rai B, Dhanireddy B, Patel FD et al. Vaginal dose, toxicity and sexual outcomes in patients of cervical cancer undergoing image based brachytherapy. Asian Pac J Cancer Prev 2014; 15: 3619-3623.

32. Rajagopalan MS, Kannan N, Kim H et al. Urethral dosimetry and toxicity with high-dose-rate interstitial brachytherapy for vaginal cancer. Brachytherapy 2013; 12: 248-253.

33. Berger D, Dimopoulos J, Georg P et al. Uncertainties in assessment of the vaginal dose for intracavitary brachytherapy of cervical cancer using a tandem-ring applicator. Int J Radiat Oncol Biol Phys 2007; 67: 1451-1459.

34. Noyes WR, Peters NE, Thomadsen BR et al. Impact of optimized treatment planning for tandem and ring, and tandem and ovoids, using high dose rate brachytherapy for cervical cancer. Int I Radiat Oncol Biol Phys 1995; 31: 79-86.

35. ICRU. International Commission of Radiation Units and Measurements. Dose and volume specification for reporting intracavitary therapy in gynecology. ICRU Report 38, Bethesda, MD, 1985.

36. Jamema SV, Kirisits C, Mahantshetty U et al. Comparison of DVH parameters and loading patterns of standard loading, manual and inverse optimization for intracavitary brachytherapy on a subset of tandem/ovoid cases. Radiother Oncol 2010; 97: 501-506

37. Kannan RA, Gururajachar JM, Ponni A et al. Comparison of manual and inverse optimisation techniques in high dose rate intracavitary brachytherapy of cervical cancer: A dosimetric study. Rep Pract Oncol Radiother 2015; 20: 365-369.

38. Chajon E, Dumas I, Touleimat $M$ et al. Inverse planning approach for 3-D MRI-based pulse-dose rate intracavitary brachytherapy in cervix cancer. Int J Radiat Oncol Biol Phys 2007; 69: 955-961. 